Изменение экспрессии некодирующих рибонуклеиновых кислот (нкРНК) играет роль в образовании различных опухолей. Синдром множественных эндокринных неоплазий 1 типа (МЭН-1) - редкое аутосомно-доминантное заболевание, обусловленное мутациями в гене $M E N 1$, кодирующем белок менин. Синдром предрасполагает к развитию опухолей околощитовидных желез, нейроэндокринных опухолей желудочно-кишечного тракта, аденом гипофиза, а также других эндокринных и неэндокринных опухолей. Механизмы образования МЭН-1-ассоциированных опухолей вследствие мутаций в гене MEN1 неясны. При отсутствии мутаций в гене MEN1 у пациентов с фенотипически схожими чертами данное состояние расценивается как фенокопии этого синдрома. Причина сочетания нескольких МЭН-1-ассоциированных опухолей у таких пациентов остается неизвестной. Возможно, что изменения в экспрессии нкРНК влияют на регуляцию сигнальных путей, в которых принимает участие менин, и могут способствовать развитию МЭН-1-ассоциированных опухолей. Идентификация даже незначительного количества агентов, взаимодействующих с менином, вносит существенный вклад в повышение уровня знаний о его патофизиологическом влиянии и способах развития опухолей в рамках синдрома МЭН-1 и его фенокопий.

КЛЮЧЕВЫЕ СЛОВА: синдром множественных эндокринных неоплазий 1 типа; менин; некодирующие PHК; микроРНК; MEN1.

\title{
THE ROLE OF NON-CODING RNAS IN THE PATHOGENESIS OF MULTIPLE ENDOCRINE NEOPLASIA SYNDROME TYPE 1
}

\author{
(c) Elizaveta O. Mamedova, Diana A. Dimitrova*, Zhanna E. Belaya, Galina A. Melnichenko
}

Endocrinology Research Centre, Moscow, Russia

Changes in the expression of non-coding ribonucleic acids (ncRNAs) take part in the formation of various tumors. Multiple endocrine neoplasia syndrome type 1 (MEN1) is a rare autosomal dominant disease caused by mutations of the MEN1 gene encoding the menin protein. This syndrome is characterized by the occurrence of parathyroid tumors, gastroenteropancreatic neuroendocrine tumors, pituitary adenomas, as well as other endocrine and non-endocrine tumors. The pathogenesis of MEN-1 associated tumors due to MEN1 mutations remains unclear. In the absence of mutations of the MEN1 gene in patients with phenotypically similar features, this condition is regarded as a phenocopy of this syndrome. The cause of the combination of several MEN-1-related tumors in these patients remains unknown. The possible cause is that changes in the expression of ncRNAs affect the regulation of signaling pathways in which menin participates and may contribute to the development of MEN-1-related tumors. The identification of even a small number of agents interacting with menin makes a significant contribution to the improvement of knowledge about its pathophysiological influence and ways of developing tumors within the MEN-1 syndrome and its phenocopies.

KEYWORDS: multiple endocrine neoplasia syndrome type 1; menin; non-coding RNAs; microRNA; MEN1.

\section{ВВЕДЕНИЕ}

Синдром множественных эндокринных неоплазий 1 типа

Синдром множественных эндокринных неоплазий 1 типа (МЭН-1) - это редкое заболевание с аутосомно-доминантным типом наследования. При данном синдроме развивается сочетанное опухолевое поражение околощитовидных желез (ОЩЖ) (90\%), желудочно-кишечного тракта (30-70\%) и аденогипофиза (30-40\%) [1]. Кроме того, в рамках синдрома могут развиваться опухоли более 20 других эндокринных и неэндокринных тканей (в том числе, около 40\% случаев - опухоли надпочечников) [1, 2]. В 1988 г. исследователи из Каролинского института в Стокгольме и университетской больницы
Уппсалы картировали ген MEN1 на длинном плече хромосомы 11q13 [3], герминальные мутации в котором приводят к развитию синдрома МЭН-1. Сам ген был идентифицирован в 1997 г. [4]. Ген MEN1 кодирует белок менин, функции которого будут рассмотрены ниже. В настоящее время описано более 1600 мутаций в данном гене, из них нонсенс-мутации составляют 23\%, миссенс - 20\%, делеции и инсерции со сдвигом рамки считывания - 41\%, делеции и инсерции без сдвига рамки считывания $6 \%$, сплайсинговые мутации - 9\%, крупные делеции $1 \%$ [5]. Семейные формы синдрома МЭН-1 составляют около 85\% случаев, а частота обнаружения спорадических форм (когда в ранее не затронутой семье выявляется один больной) наблюдаются значительно реже (около 15\% случаев) [5]. 
Гипотеза «двойного удара» Кнудсона может объяснять, почему ген MEN1 является геном-супрессором опухолевого роста [6]. В 1971 г. А. Кнудсон предложил гипотезу, объясняющую механизм возникновения наследственной и спорадической форм ретинобластомы. Он предположил, что для возникновения опухоли в клетке должны произойти две последовательные мутации: первая мутация - в клетках зародышевой линии, а вторая мутация - соматическая. При ненаследственной форме должны возникнуть две мутации, причем в одной и той же соматической клетке. Это снижает вероятность такого совпадения, и поэтому спорадическая ретинобластома как результат двух соматических мутаций наблюдается в более зрелом возрасте [6]. У пациентов с герминальными мутациями в гене MEN1 потеря гетерозиготности (LOH) на хромосоме 11q13 обнаруживается в более чем 90\% опухолей, тогда как в спорадических аналогах этих эндокринных опухолей LOH 11q13 наблюдается в 5-50\% случаев [7]. Например, биаллельные соматические мутации в гене MEN1 в спорадических аденомах ОЩЖ были выявлены в 12-35\% случаев [8]. Соматические мутации в гене MEN1 в спорадических опухолях поджелудочной железы (ПЖ) обнаруживаются примерно в 25-44\% [9, 10]. В спорадических аденомах гипофиза процент соматических мутаций гена MEN1 низок и составляет примерно 2-5\% [11, 12], в опухолях надпочечника - менее $5 \%$ [13].

В 10-30\% семейных случаев МЭН-1 и 60-80\% спорадических случаев синдрома мутации в гене MEN1 не выявляются, что может быть связано с крупными делециями данного гена, мутациями в промоторе или других нетранслируемых областях, которые обычно не анализируются в «рутинных» генетических тестах $[5,14]$. Известно, что гиперметилирование сайтов CpG в промоторных областях генов-супрессоров опухолей может приводить к потере функции этих генов [15]. Так, в тканях аденом ОЩЖ наблюдалось гиперметилирование промоторной области гена MEN1 на участках островков CpG 24-31 у пациентов с МЭН-1, и от частоты метилирования зависела тяжесть клинических проявлений заболевания [16]. Кроме того, развитие МЭН-1-ассоциированных опухолей у таких пациентов может быть обусловлено другими причинами: мутациями в других, еще не установленных, генах, эпигенетическими изменениями, а также, вероятно, случайным сочетанием нескольких опухолей у одного пациента $[1,17,18]$. Подробная информация о фенокопиях синдрома МЭН-1 представлена в нашем обзоре [19].
Белок менин и его функции

Белок менин состоит из 610 аминокислотных остатков, последовательность которых не гомологична ни одному известному белку. Менин экспрессируется во всех органах и тканях, однако его экспрессия варьирует в зависимости от типа ткани [20]. На клеточном уровне в основном он находится в ядре, в небольшом количестве также может быть обнаружен в цитоплазме и клеточной мембране. Менин подвергается посттрансляционным изменениям, таким как фосфорилирование (по аминокислотным остаткам Ser394, Thr397, Thr399, Ser487, Ser543, Ser583), сумоилирование, пальмитирование. В структуре менина выделяют два основных сигнала ядерной локализации (nuclear localization signals, NLS) — NLS1 (аминокислотные остатки 479-497) и NLS2 (аминокислотные остатки 588-608), а также третий, дополнительный, NLS (NLSa, аминокислотные остатки 546-572) [21]. При герминальных и соматических мутациях в гене MEN1, приводящих к укорочению белка (нонсенс-мутации и мутации со сдвигом рамки считывания) и потере одного или обоих основных NLS, происходит инактивация белка. При миссенс-мутациях синтезируемый белок подвергается деградации протеасомами, что предотвращает его функциональную активность. Менин не обладает ферментативной активностью [21].

В исследованиях было показано, что менин взаимодействует со множеством белков (более 50) в составе различных белковых комплексов. В целом белки, взаимодействующие с менином, можно разделить на четыре большие группы: 1) активаторы транскрипции; 2) ингибиторы транскрипции; 3) белки, участвующие в передаче сигналов и 4) прочие белки с разнообразными функциями (например, регулирующие репарацию ДНК, клеточный цикл, поддерживающие структуру клетки и др.) $[20,22]$. Белки, взаимодействующие с менином, перечислены в таблице 1.

Менин воздействует на некоторые сигнальные пути (табл. 2). Кроме того, регуляция самого менина осуществляется различными белками и сигнальными путями, в том числе теми, на которые воздействует и он сам (табл. 3). Взаимодействуя с различными белковыми комплексами, менин может принимать участие в эпигенетической регуляции [37, 38].

Каким образом мутации в гене MEN1, приводящие, соответственно, к синтезу дефектного белка менина, приводят к образованию специфических опухолей, остается неясным.

Таблица 1. Белки, взаимодействующие с менином [20, 21].

Активаторы и ингибиторы транскрипции

c-MYB, MLL1, PEM, RUNX2, DAXX, HDACs mSIN3A, LEDGF, PRMT5, SUV39H1, DNMT1, FBP1, FOXA2, HLXB9/MNX1, JUND, c-MYC; NFkB - p50, p52, р65; ядерные рецепторы (AR, ERa, LXRa, PPARa, PPARy, RXR, VDR); SMADs (SMAD1, SMAD3, SMAD5), SIRT1, SON, TCF3, TCF4, $\beta$-катенин; изоформы RNA-Pol-II (pSer5, pSer2); SKIP

Белки сигнальных путей

AKT1, FOXO1, NM23 $\beta$, GRB2, RAS, SOS1

Другие белки

RPA2, ASK, CHES1, FANCD2, GFAP, Виментин, NMMHC-IIA, IQGAP1, ARS2, CHIP,

HSP70 
Таблица 2. Сигнальные пути, регулирующиеся менином.

\begin{tabular}{lcc}
\hline \multicolumn{1}{c}{ Название сигнального пути } & Влияние менина & Источники \\
\hline TGF $\beta$ (трансформирующий фактор роста бета) & $\uparrow$ & {$[23]$} \\
BMP (костный морфогенетический белок) & $\uparrow \downarrow$ & {$[24]$} \\
Wnt & $\uparrow$ & {$[25]$} \\
Nuclear receptor (ядерные рецепторы) & $\uparrow$ & {$[26,27,28]$} \\
Ras (малые G-белки) & $\downarrow$ & {$[29,30]$} \\
PI ${ }_{3}$ K/Akt (протеин киназа B) и FOXO & $\downarrow$ & {$[31,32]$} \\
Hedgehog & $\downarrow$ & {$[33]$} \\
\hline
\end{tabular}

Примечание: $\downarrow$ - ингибирует; $\uparrow$ активирует.

Таблица 3. Белки и сигнальные пути, регулирующие экспрессию менина.

\begin{tabular}{lcc}
\hline \multicolumn{1}{c}{ Название сигнального пути } & Влияние на менин & Источники \\
\hline Пролактин и его сигнальные пути & $\downarrow$ & {$[34]$} \\
TGFß (трансформирующий фактор роста бета) & $\uparrow$ & {$[24]$} \\
Соматостатиновый сигнальный путь & $\uparrow$ & {$[35]$} \\
PI K/Akt сигнальный путь & $\downarrow$ & {$[36]$} \\
K-Ras-индуцированное метилирование ДНК & $\downarrow$ & {$[29]$} \\
\hline
\end{tabular}

Примечание: $\downarrow$ - ингибирует; $\uparrow$ активирует.

\section{НЕКОДИРУЮЩИЕ РНК ПРИ МЭН-1}

Некодирующие РНК (нкРНК) не имеют открытых рамок считывания и поэтому, как следует из их названия, не кодируют белки. МикроРНК (miR) относятся К коротким нкРНК и состоят из 20-24 пар нуклеотидов. МикроРНК ингибируют экспрессию генов посредством двух механизмов: комплементарного связывания ДНК в хроматине, что приводит к РНК-индуцированному подавлению транскрипции генов, или комплементарного связывания матричной РНК (мРНК), что приводит к ее деградации и блокированию трансляции [38]. Гены, кодирующие микроРНК, составляют 1-5\% генома человека и контролируют экспрессию тысяч мРНК, причем несколько микроРНК могут принимать участие в экспрессии одной мРНК [39]. Длинные нкРНК (днкРНК) являются транскриптами длиной около 200 и более пар нуклеотидов, которые могут взаимодействовать с ДНК и белками, посредством чего они участвуют в эпигенетической регуляции [40].

Изменение экспрессии микроРНК считается важным событием в инициации и прогрессировании опухолеобразования, и уже имеется большое количество данных, свидетельствующих о его патогенетической значимости. Так, в литературе описаны различия в экспрессии микроРНК между нормальными тканями, доброкачественными и злокачественными опухолями гипофиза [41], ОЩж [42] и коры надпочечника [43].

На мРНК менина влияют различные микроРНК. Кроме того, имеются данные, указывающие на то, что менин как фактор транскрипции участвует в синтезе микроРНК (см. ниже раздел «Менин и микроРНК»). Таким образом, изменения в эпигенетической регуляции сигнальных путей при синдроме МЭН-1 посредством микроРНК могут способствовать развитию опухолевого процесса.

\section{Менин и микроРНК}

Предположения о том, что экспрессия микроРНК может контролироваться транскрипционным фактором/ транскрипционными факторами или другими микроРНК, образуя петли обратной связи, либо же, наоборот, микроРНК совместно с транскрипционными факторами регулируют экспрессию генов-мишеней, образуя петли прямой связи, были выдвинуты достаточно давно [44]. В исследовании Luzi и соавт. было продемонстрировано, что miR-24-1 непосредственно связывается с 3'-нетранслируемой областью (3'-UTR) мРНК менина и подавляет его экспрессию [45]. При этом также было показано, что miR-24-1 экспрессируется только в LOH-отрицательных аденомах ОЩж пациентов с генетически подтвержденным синдромом МЭН-1 (с сохранным аллелем дикого типа) и не экспрессируется в LOH-положительных аденомах, что говорит о том, что менин необходим для экспрессии этой микроРНК. Несмотря на остаточную экспрессию мPHK в LOH-отрицательных аденомах ОЩЖ пациентов с МЭН-1 (за счет оставшегося аллеля дикого типа), по сравнению с LOH-положительными аденомами, где экспрессия мPHК гена MEN1 отсутствовала, экспрессия самого менина отсутствовала в обоих подтипах, что свидетельствовало о том, что гиперэкспрессия miR-24-1 негативно влияет на мРНК менина. Таким образом, авторы предположили наличие отрицательной обратной связи, по которой менин необходим для экспрессии miR24-1, при этом последняя подавляет экспрессию менина в отсутствие LOH второго аллеля гена MEN1, то есть «выключает» этот аллель, что согласуется с теорией Кнудсона [45]. В дальнейшем Vijayaraghavan и соавт. установили, что miR-24 непосредственно снижает экспрессию менина в клеточных линиях MIN6 (клетки инсулиномы мыши) и ßlox5 (иммортализированные $\beta$-клетки человека), а также подтвердили наличие петли отрицательной обратной 
связи между менином и miR-24 [46]. Кроме того, в этой работе было показано, что снижение экспрессии менина под воздействием miR-24 приводит к снижению экспрессии ингибиторов клеточного цикла р27 $7^{\mathrm{kip} 1}$ и p18 $18^{\text {ink4c }}$ [46]. В работе Ehrlich и соавт. было продемонстрировано, что экспрессия miR-24 повышена в клеточных линиях холангиокарциномы человека и что данная микроРНК подавляет экспрессию менина [47]. В другом исследовании Luzi и соавт. было показано, что менин непосредственно связывается с первичной последовательностью РНК предшественника этой микроРНК (pri-miR-24-1) и способствует образованию miR-24-1 [48].

Кроме miR-24, были найдены некоторые другие микроРНК, способные подавить экспрессию менина В различных тканях. В еще одном исследовании Luzi и соавт. было показано, что менин, взаимодействуя с промотором гена miR-26a, индуцирует экспрессию этой микроРНК. «Выключение» МРНК менина приводит к снижению экспрессии miR-26a [49]. MiR-26a, в свою очередь, является регулятором SMAD1 белка, играющего важную роль в клеточном цикле и росте [49]. B работе Li и соавт. было выявлено, что по сравнению со здоровой тканью в тканях нейробластом повышена экспрессия miR-421, что способствует пролиферации, миграции и инвазии ее клеток [50]. На клеточных линиях нейробластомы SHSY5Y, SHEP и IMR-32 было показано, что менин является мишенью miR-421, которая, связываясь с 3'-UTR его мРНК, подавляет его экспрессию. При этом в клеточной линии нейробластомы человека SHSY5Y увеличение концентрации менина приводило к нивелированию эффектов сверхэкспрессии miR-421 [50]. B работе Lu и соавт. на клеточной линии MIN6 было выявлено, что miR-17, экспрессия которой повышается под воздействием высоких уровней глюкозы в $\beta$-клетках ПЖ, напрямую подавляет экспрессию менина посредством связывания с 3'-UTR его мРНК, тем самым способствуя пролиферации $\beta$-клеток ПЖ [51]. Отрицательная корреляция между miR-762 и менином была обнаружена в исследовании Hou и соавт. в тканях рака яичника. Выявлено, что miR-762 может непосредственно подавлять экспрессию менина, связываясь с 3'-UTR его мРНК, активируя сигнальный путь Wnt/ $\beta$-катенин, и способствовать тем самым пролиферации и метастазированию раковых клеток яичников [52]. В исследовании Gurung и соавт. выявлено, что менин взаимодействует с белком ARS2, компонентом ядерного САР-связывающего комплекса, имеющим решающее значение для синтеза некоторых микроРНК, и повышает процессинг pri-let-7a и pri-miR-155 в prelet-7a и pre-miR-155 соответственно, не влияя на уровень самих предшественников [53]. Мишенью микроРHK let-7а является белок IRS2, который играет важную роль в передаче сигналов инсулина и индуцированной инсулином пролиферации клеток ПЖЖ. Эти результаты раскрывают механизм, посредством которого менин подавляет пролиферацию клеток, по крайней мере частично, путем стимулирования процессинга микроРНК let-7a [53]. В работе Ouyang и соавт. продемонстрировано, что miR-29 подавляет экспрессию менина в клеточной линии эпителия кишечника крысы [54]. Данные о взаимном влиянии различных микроРНК и менина представлены в таблице 4.
Исследования, посвященные оценке экспрессии микроРНК в опухолях при синдроме МЭН-1, немногочисленны. В работе Luzi и соаBт. проводилось сравнение экспрессии микроРНК с помощью микрочипов в семи аденомах ОЩЖ пациентов с генетически подтвержденным синдромом МЭН-1 (из них в четырех аденомах была выявлена LOH в локусе 11q13, тогда как в трех аденомах аллель дикого типа был сохранен) с двумя спорадическими аденомами ОЩж (без соматических мутаций в MEN1) и двумя образцами тканей здоровых ОЩЖ (использовался свежезамороженный материал) [55]. Было выявлено, что экспрессия восьми микроРНК (hsa-miR-4258, hsa-miR-664, hsa-miR-299-5p, hsa-miR-625, hsa-miR-877-5p, hsa-miR-3614-5p, hsa-miR-23c, hsa-miR-3938) отличалась между LOH-отрицательными ОЩж и контролем, экспрессия двух микроРHK (hsa-miR-1301, hsa-miR-664) отличалась между LOH-положительными аденомами ОЩЖ и контролем. Экспрессия шести микроРHK (hsa-miR-4258, hsa-miR-1301, hsa-miR-485-5p, hsa-miR-3944, hsa-miR-135b, hsa-miR-1261) отличалась между LOH-положительными и LOH-отрицательными аденомами ОЩж. При этом примечательна различная экспрессия трех микроРНК (miR-4258, miR-664 и miR-1301) в LOH-положительных и LOH-отрицательных аденомах ОЩЖ пациентов с МЭН-1. Так, экспрессия miR-4258 была подавлена в LOH-положительных аденомах ОЩЖ по сравнению с LOH-отрицательными аденомами ОЩЖ, что демонстрирует необходимость наличия хотя бы одного аллеля дикого типа для экспрессии этой микроРНК. Экспрессия miR-4258 была выше в LOH-отрицательных аденомах ОЩЖ по сравнению с контролем. Экспрессия miR-664 была выше в LOHотрицательных аденомах и ниже в LOH-положительных аденомах по сравнению с контролем. Экспрессия miR-1301 была выше в LOH-положительных аденомах ОЩЖ по сравнению с LOH-отрицательными ОЩЖ и контролем. Таким образом, авторы делают вывод, что для экспрессии некоторых микроРНК необходимо наличие хотя бы одного аллеля дикого типа, кодирующего белок менин, а также что miR-4258, miR-1301 и miR-664 являются наилучшими прогностическими и диагностическими маркерами в разграничении МЭН-1-ассоциированных аденом ОЩЖ, спорадических аденом ОЩЖ и здоровых ОЩЖ, а также в разграничении между МЭН-1-ассоциированными аденомами ОЩЖ с или без LOH в локусе $11 q 13$ [55]. В этой же работе авторы провели поиск возможных генов-мишеней, известных в патогенезе образования опухолей ОЩЖ, для выявленных микроРНК с измененной экспрессией с помощью компьютерного алгоритма ComiR tool. В частности, miR-4258 подавляет экспрессию гена CCND1, кодирующего циклин D1 (положительный регулятор прогрессии клеточного цикла). Таким образом, снижение экспрессии miR-4258, следующее за потерей аллеля дикого типа гена MEN1, может быть ответственно за индукцию неконтролируемого роста клеток ОЩЖ. Повышенная экспрессия miR-1301 при потере аллеля дикого типа гена MEN1 подавляет экспрессию генов CDKN1B, RB1, CTNNB1 и RET. MiR-664 снижает экспрессию гена $C D K N 2 C$ и гена-супрессора опухолей $C D C 73$, кодирующего парафибромин [55].

В исследовании Grolmusz и соавт. проводилось сравнение 16 образований ОЩЖ от пациентов с генетически подтвержденным синдромом МЭН-1 и 40 спорадических 
Таблица 4. Взаимное влияние менина и микроРНК.

МикроРНК, которые влияют на менин

\begin{tabular}{|c|c|c|c|}
\hline $\begin{array}{l}\text { Название } \\
\text { микроРНК }\end{array}$ & Влияние & Характер взаимодействия & $\begin{array}{c}\text { Биологический объект } \\
\text { исследования, ссылка } \\
\text { на источник }\end{array}$ \\
\hline $\mathrm{miR}-24-1^{\S}$ & $\downarrow$ экспрессию & Связывается с 3’UTR мPHК менина & BON1 [45] \\
\hline $\mathrm{miR}-24^{\mathrm{s}}$ & $\downarrow$ экспрессию & Связывается с 3'UTR мРНК менина & MIN6, $\beta$ lox5 [46] \\
\hline miR-24 & $\downarrow$ экспрессию & Корреляция уровней экспрессии & $\begin{array}{c}\text { Mz-ChA-1, TFK-1, } \\
\text { SG231, CCLP-1, HuCC-T1, } \\
\text { HuH-28 [47] }\end{array}$ \\
\hline $\operatorname{miR}-421$ & $\downarrow$ экспрессию & Связывается с 3’UTR мPHК менина & $\begin{array}{l}\text { SHSY5Y, SHEP и IMR-32 } \\
{[50]}\end{array}$ \\
\hline miR-17 & $\downarrow$ экспрессию & Связывается с 3'UTR мPHК менина & MIN6 [51] \\
\hline miR-762 & $\downarrow$ экспрессию & Связывается с 3'UTR мPHК менина & SKOV3 [52] \\
\hline miR-29b & $\downarrow$ экспрессию & $\begin{array}{l}\text { Связывается с единичным сайтом } \\
\text { кодирующей области мРНК менина }\end{array}$ & IECs [54] \\
\hline
\end{tabular}

МикроРНК, на которые влияет менин

\begin{tabular}{|c|c|c|c|}
\hline $\begin{array}{l}\text { Название } \\
\text { микроРНК }\end{array}$ & Влияние & Характер взаимодействия & $\begin{array}{c}\text { Биологический объект } \\
\text { исследования, ссылка } \\
\text { на источник }\end{array}$ \\
\hline
\end{tabular}

\begin{tabular}{|c|c|c|c|}
\hline $\operatorname{miR}-24^{\S}$ & 个 экспрессию & $\begin{array}{l}\text { Увеличение экспрессии } \\
\text { при гиперэкспрессии менина }\end{array}$ & MIN6, $\beta$ lox5 [46] \\
\hline $\operatorname{miR}-24-1^{\S}$ & 个 экспрессию & $\begin{array}{l}\text { Менин связывается с РНК } \\
\text { предшественника этой микроРНК } \\
\text { pri-miR-24-1 }\end{array}$ & BON1 [48] \\
\hline
\end{tabular}

miR-26a $\quad$ экспрессию

Снижение экспрессии этой микроРНК

при «сайленсинге» мРНК менина. hADSCs [49]

Менин связывается с промотором гена

miR-26а и индуцирует его экспрессию

\begin{tabular}{lll}
\hline let-7a* & $\uparrow$ процессинг pri-miR & \\
& в pre-miR, увеличивая & Уровни let-7a и miR-155 были снижены \\
количество зрелой & при эксцизии гена Men1 в клеточной \\
& $\begin{array}{l}\text { микроРНК. Не влияет } \\
\text { на уровень pri-miR }\end{array}$ & линии \\
&
\end{tabular}

Перечисленные клеточные линии: BON1 - клеточная линия нейроэндокринных опухолевых клеток Пж человека; МIN6 - клеточная линия инсулиномы мыши; ßlox5 - иммортализованные $\beta$-клетки Пж человека; Mz-ChA-1, TFK-1, SG231, CCLP-1, HuCC-T1, НuH-28 - клеточные линии холангиокарциномы человека; SHSY5Y, SHEP и IMR-32 - клеточные линии нейробластомы; SKOV3 - клеточная линия аденокарциномы яичника человека; IECs - клетки эпителия кишечника крысы; hADSCs - человеческие стволовые клетки, выделенные из жировой ткани (в данном случае, индуцированные по пути дифференцировки в остеобласты); MEFs - мышиные эмбриональные фибробласты.

$\downarrow$ - снижает, $\uparrow$ - повышает.

$\S$ - miR-24 транскрибируется с хромосомы 9 (miR-24-1) и с хромосомы 19 (miR-24-2), обе miR-24 (miR-24-1 и miR-24-2) идентичны по строению и отличаются только хромосомой происхождения [из статьи 46].

* - влияет на уровень зрелой микроРНК, но не на уровень ее предшественника (см. в тексте).

образований ОЩЖ [56]. Наличие сохранного аллеля дикого типа гена MEN1 определялось с помощью иммуногистохимического (ИГХ) исследования, по результатам которого окраска ядер клеток на менин отсутствовала во всех МЭН-1-ассоциированных образованиях ОЩЖ и в 28\% (11/40) спорадических образований ОЩЖ. При исследовании соматических мутаций в тканях спорадических образований ОЩЖ в 25\% случаев (10/40) были выявлены мутации в гене MEN1. Таким образом, авторы рассчитали чувствительность (86\%) и специфичность (87\%) использования ИГХ-метода для определения соматических мутаций. Анализ экспрессии микроРНК проводился из материала, выделенного из парафиновых блоков, методом количественной ПЦР в реальном времени для следующих микроРHK: hsa-miR-24, hsa-miR-28, hsa-miR-326, hsa-miR-484, hsa-miR-637, hsa-miR-744, при этом экспрессию hsa-miR-637 не удалось обнаружить во всех образцах. По остальным микроРНК не было выявлено значимых различий в менин-положительных и менин-отрицательных тканях образований ОЩЖ, независимо от наличия или отсутствия герминальной мутации в гене MEN1. Однако экспрессия hsa-miR-24 
и hsa-miR-28 была выше в спорадических образованиях ОЩЖ по сравнению с МЭН-1-ассоциированными образованиями. Кроме того, при дальнейшем делении группы спорадических образований ОЩж на менин-положительные и менин-отрицательные, в обеих группах экспрессия этих микроРНК была выше, чем в МЭН-1-ассоциированных образованиях [56].

В исследовании Lines и соавт. изучалась экспрессия miR-15a, miR-16-1 и let-7а в аденомах гипофиза у мышей с гетерозиготным нокаутом гена Men 1 [57]. Экспрессия всех трех микроРНК была значимо подавлена по сравнению с контрольной группой (здоровые мышиные гипофизы). Также в исследовании было показано, что экспрессия мPHK гена Ccnd1 и белка циклина D1 была значимо повышена в аденомах гипофиза Men 1+/- мышей по сравнению со здоровыми мышиными гипофизами. Была выявлена обратная корреляция между уровнями miR-15a и miR-16-1 и мPHK Ccnd1, что может свидетельствовать о регуляции циклина D1 этими микроРНК. Это предположение было подтверждено на клеточных культурах, когда введение антагонистов к miR-15a и miR-16-1 приводило к значимому повышению экспрессии циклина D1 [57]. Анализ экспрессии мРНК мишени микроРHK let-7a - Kras - выявил значимое повышение экспрессии Kras в аденомах гипофиза Men1+/- мышей, однако значимой обратной корреляции между экспрессией Kras и let-7а не наблюдалось. В данной работе авторы также выявили, что отсутствие экспрессии менина в клеточной культуре приводит к снижению экспрессии miR-15a, но не miR-16-1. Кроме того, miR-15a и miR-16-1 непосредственно не влияют на экспрессию менина, что говорит об отсутствии петель обратной связи в данном случае [57].

В настоящее время продолжается поиск микроРНК, влияющих на менин и его функции. В своей работе Nagy и соавт. провели анализ литературы и сравнили данные о достоверно отличающейся экспрессии микроРНК в здоровых тканях и опухолях (доброкачественных и злокачественных) гипофиза, ОЩЖ и коры надпочечников и отметили изменения в экспрессии микроРНК, которые принадлежат к одному из крупнейших кластеров микроРНК в геноме человека - DLK1-MEG3. МикроРНК этого кластера регулируют сигнальные пути, которые часто вовлечены в опухолевый генез, например, mTOR, MAPK, Wnt/ $\beta$-катенин, p53, в которых также участвует белок менин. Однако экспериментальные данные о потенциальной связи между микроРHК кластера DLK1-MEG3 и MEN1 отсутствуют [58]. Другую потенциальную связь между MEN1 и микроРHK Nagy и соавт. в своей статье предположили с геном микроРНK miR-142-3р, который регулируется белком менином в тканях остеосаркомы человека [58]. Известно, что адренокортикотропный гормон индуцирует экспрессию микроРНК miR-142-3р, которая, в свою очередь, воздействует на глюкокортикоидные рецепторы в надпочечниках. Также имеются данные о том, что экспрессия глюкокортикоидного рецептора альфа повышена в адренокортикальных аденомах, продуцирующих кортизол, по сравнению с гормонально-неактивными аденомами и здоровыми тканями [59]. Таким образом, была выдвинута гипотеза о регуляторной петле, способствующей онкогенезу в тканях надпочечника при отсутствии белка менина: снижение экспрессии менина в адренокортикальных опухолях привело бы к снижению продукции микроРHK miR-142-3р и тем самым - к увеличению количества глюкокортикоидных рецепторов, вызывая образование опухолей. Для подтверждения этого потенциального пути авторы предполагают провести исследования, чтобы продемонстрировать регуляцию экспрессии miR-142-3р менином в тканях коры надпочечников [58].

\section{Менин и днкРНК}

Известно, что некоторые днкРНК содержатся в хроматин-ремоделирующих белковых комплексах, которые могут подавлять экспрессию генов $[60,61]$. О взаимодействии днкРНК и менина в литературе имеются лишь скудные данные. Так, Modali и соавт. в своем исследовании охарактеризовали эпигенетическую регуляцию днкРНК Meg3 менином в $\beta$-клетках ПЖ и идентифицировали протоонкоген с-Mеt (рецептор фактора роста гепатоцитов) как ген-мишень Meg3 [62]. На мышиной клеточной культуре инсулиномы MIN6 было выявлено, что менин активирует днкРHК Meg3, вызывая увеличение экспрессии этой днкРНК путем триметилирования гистона Н3 по лизину 4 и гипометилирования CPG в CRE-сайте промотора гена Meg3. При отсутствии белка менина этого не происходило. Повышенная экспрессия днкPHК Meg3 вызывала снижение экспрессии протоонкогена c-Met, что приводило к подавлению активности опухолевых клеток в MIN6. Это же было доказано при сравнении клеток ПЖЖ у мышей дикого типа и мышей Men 1+/- (достоверное снижение экспрессии днкРНК Meg3 в клетках опухолей у мышей Men 1+/- и, соответственно, усиленное окрашивание на с-Met по сравнению с нормальными $\beta$-клетками в том же срезе ПЖ). Регуляция MEG3 и с-MET была дополнительно оценена в замороженных образцах опухолей $\beta$-клеток ПЖЖ пациентов с мутациями в MEN1 и без них: в четырех из пяти образцов с мутацией в MEN1 и во всех трех без мутации было выявлено значимое снижение экспрессии днкPHK MEG3, и, в свою очередь, во всех пяти опухолях ПЖЖ с мутацией и в двух из трех опухолей ПЖЖ без мутации отмечено значимое увеличение экспрессии белка с-МЕТ. Интересно, что экспрессия MEG3 и с-MET также изменялась в спорадических инсулиномах человека с гиперметилированием по CRE-сайту промотора MEG3, как и у пациентов с мутацией в гене MEN1 [62].

\section{ЗАКЛЮЧЕНИЕ}

Таким образом, исследования последних лет приоткрыли механизмы, по которым развиваются опухоли при выпадении функции гена MEN1 вследствие его мутаций, а также вероятные эпигенетические механизмы «выключения» гена MEN1, что может объяснять как развитие МЭН-1-ассоциированных опухолей, так и развитие фенокопий МЭН-1 синдрома. Так, было показано, что менин регулирует экспрессию ряда нкРНК, которые, в свою очередь, регулируют транскрипцию генов, кодирующих факторы, влияющие на клеточную пролиферацию. Соответственно, это может быть одним из механизмов образования опухолей при мутациях в гене MEN1. Кроме того, есть ряд нкРНК, которые регулируют экспрессию менина, и нарушения в экспрессии этих нкРНК, в свою 
очередь, могут объяснять выпадение функции менина без выявленной мутации в гене MEN1. Конечно, нельзя полностью исключить то, что могут существовать еще не выявленные мутации в других генах или может иметь место случайное сочетание нескольких опухолей при развитии фенокопий МЭН-1 синдрома. Идентификация любых агентов, взаимодействующих с менином, вносит существенный вклад в повышение уровня знаний о его патофизиологическом влиянии и способах развития опухолей в рамках синдрома МЭН-1. Менин участвует в эпигенетических процессах, и его потеря может приводить к эпигенетическим изменениям, способствующим образованию опухолей. Учитывая, что эпигенетические изменения могут быть обратимы, воздействуя на них, можно вернуть эпигеном в исходное (нормальное) состояние. Поэтому в настоящее время выдвигаются предположе- ния о создании таргетных препаратов для коррекции эпигенетических изменений. Исследования сложных сетей молекулярного пути менина будут полезны для разработки новых терапевтических методов лечения синдрома МЭН-1.

\section{ДОПОЛНИТЕЛЬНАЯ ИНФОРМАЦИЯ}

Источник финансирования. Поисково-аналитическая работа и подготовка статьи проведены за счет гранта Российского научного фонда (проект № 19-15-00398).

Конфликт интересов. Авторы декларируют отсутствие явных и потенциальных конфликтов интересов, связанных с публикацией настоящей статьи.

Участие авторов. Все авторы внесли значимый вклад в написание статьи, прочли и одобрили финальный вариант рукописи до публикации.

\section{СПИСОК ЛИТЕРАТУРЫ | REFERENCES}

1. Thakker R, Newey P, Walls G, et al. Clinical practice guidelines for multiple endocrine neoplasia Type 1 (MEN1). J Clin Endocrinol Metab. 2012;97(9):2990-3011. doi: 10.1210/jc.2012-1230.

2. Рожинская Л.Я., Хандаева П.М., Луценко А.С., и др. Рецидив аденомы гипофиза с изменением гормональной активности у пациентки с синдромом множественной эндокринной неоплазии 1-го типа // Альманах клинической медицины. 2018. — T.46. — №3. - C. 270-275. [Rozhinskaya LY,

Khandaeva PM, Lutsenko AS, et al. Relapse of the pituitary adenoma with a change of its hormonal activity in a female patient with multiple endocrine neoplasia syndrome type 1. Almanac of Clinical Medicine. 2018;46(3):270-275. (In Russ).] doi: 10.18786/2072-0505-2018-46-3-270-275.

3. Larsson C, Skogseid B, Oberg K, et al. Multiple endocrine neoplasia type 1 gene maps to chromosome 11 and is lost in insulinoma. Nature. 1988;332(6159):85-87. doi: 10.1038/332085a0.

4. Chandrasekharappa SC, Guru SC, Manickam P, et al. Positional cloning of the gene for multiple endocrine neoplasia-type 1. Science. 1997;276(5311):404-407. doi: 10.1126/science.276.5311.404.

5. Falchetti A. Genetics of multiple endocrine neoplasia type 1 syndrome: what's new and what's old. F1000Res. 2017;6(73):F1000 Faculty Rev-73. doi: 10.12688/f1000research.7230.1.

6. Knudson AG Jr. Mutation and cancer: statistical study of retinoblastoma. Proc Natl Acad Sci U S A. 1971;68(4):820-823. doi: 10.1073/pnas.68.4.820.

7. Lemos MC, Thakker RV. Multiple endocrine neoplasia type 1 (MEN1): analysis of 1336 mutations reported in the first decade following identification of the gene. Hum Mutat. 2008;29(1):22-32. doi: 10.1002/humu.20605.

8. Costa-Guda J, Arnold A. Genetic and epigenetic changes in sporadic endocrine tumors: parathyroid tumors. Mol Cell Endocrinol. 2014;386(1-2):46-54. doi: 10.1016/j.mce.2013.09.005

9. Jiao Y, Shi C, Edil BH, et al. DAXX/ATRX, MEN1, and mTOR pathway genes are frequently altered in pancreatic neuroendocrine tumors. Science. 2011;331(6021):1199-1203. doi: 10.1126/science.1200609.

10. Corbo V, Dalai I, Scardoni M, et al. MEN1 in pancreatic endocrine tumors: analysis of gene and protein status in 169 sporadic neoplasms reveals alterations in the vast majority of cases. Endocr Relat Cancer. 2010;17(3):771-783. doi: 10.1677/ERC-10-0028.

11. Karhu A, Aaltonen LA. Susceptibility to pituitary neoplasia related to MEN-1, CDKN1B and AIP mutations: an update. Hum Mol Genet. 2007;16 Spec No 1:R73-79. doi: 10.1093/hmg/ddm036.

12. Evans CO, Brown MR, Parks JS, et al. Screening for MEN1 tumor suppressor gene mutations in sporadic pituitary tumors. J Endocrinol Invest. 2000;23(5):304-309. doi: 10.1007/BF03343727.

13. Görtz B, Roth J, Speel EJ, et al. MEN1 gene mutation analysis of sporadic adrenocortical lesions. Int J Cancer. 1999;80(3):373-379. doi: 10.1002/(sici)1097-0215(19990129)80:3<373::aid-ijc7>3.0.co;2-b.

14. Falchetti A, Brandi ML. Multiple endocrine neoplasia type I variants and phenocopies: more than a nosological issue? J Clin Endocrinol Metab. 2009;94(5):1518-1520. doi: 10.1210/jc.2009-0494.
15. Esteller M. Epigenetics in cancer. N Engl J Med 2008;358(11):1148-1159. doi: 10.1056/NEJMra072067.

16. De Paoli-Iseppi R, Prentice L, Marthick JR, et al. Multiple endocrine neoplasia type 1: clinical correlates of MEN1 gene methylation. Pathology. 2018;50(6):622-628. doi: 10.1016/j.pathol.2018.05.006.

17. Alrezk R, Hannah-Shmouni F, Stratakis CA. MEN4 and CDKN1B mutations: the latest of the MEN syndromes. Endocr Relat Cancer. 2017;24(10):T195-T208. doi: 10.1530/ERC-17-0243.

18. Turner JJ, Christie PT, Pearce SH, et al. Diagnostic challenges due to phenocopies: lessons from Multiple Endocrine Neoplasia type1 (MEN1). Hum Mutat. 2010;31(1):E1089-1101. doi: 10.1002/humu.21170.

19. Мамедова Е.О., Мокрышева Н.Г., Пржиялковская Е.Г., и др. Варианты и фенокопии синдрома множественных эндокринных неоплазий 1 типа // Терапевтический архив. - 2014. - T.86. №10. - C. 87-91. [Mamedova EO, Mokrysheva NG, Przhiialkovskaia EG, et al. Multiple endocrine neoplasia type 1 variants and phenocopies. Ter Arkh. 2014;86(10):87-91. (In Russ).]

20. Matkar S, Thiel A, Hua X. Menin: a scaffold protein that controls gene expression and cell signaling. Trends Biochem Sci. 2013;38(8):394-402. doi: 10.1016/j.tibs.2013.05.005

21. Agarwal SK. The future: genetics advances in MEN1 therapeutic approaches and management strategies. Endocr Relat Cancer. 2017;24(10):T119-T134. doi: 10.1530/ERC-17-0199.

22. Dreijerink KM, Timmers HT, Brown M. Twenty years of menin: emerging opportunities for restoration of transcriptional regulation in MEN1. Endocr Relat Cancer. 2017;24(10):T135-T145. doi: 10.1530/ERC-17-0281.

23. Kaji H, Canaff L, Lebrun JJ, et al. Inactivation of menin, a Smad3interacting protein, blocks transforming growth factor type beta signaling. Proc Natl Acad Sci U S A. 2001;98(7):3837-3842. doi: 10.1073/pnas.061358098.

24. Kaji H. Menin and bone metabolism. J Bone Miner Metab. 2012:30(4):381-387. doi: 10.1007/s00774-012-0355-3.

25. Chen G, Wang M, Farley S, et al. Menin promotes the Wnt signaling pathway in pancreatic endocrine cells. Mol Cancer Res. 2008;6(12):1894-907. doi: 10.1158/1541-7786.MCR-07-2206.

26. Imachi H, Murao K, Dobashi H, et al. Menin, a product of the MENI gene, binds to estrogen receptor to enhance its activity in breast cancer cells: possibility of a novel predictive factor for tamoxifen resistance. Breast Cancer Res Treat. 2010;122(2):395-407. doi: 10.1007/s10549-009-0581-0.

27. Dreijerink KM, Varier RA, van Beekum O, et al. The multiple endocrine neoplasia type 1 (MEN1) tumor suppressor regulates peroxisome proliferator-activated receptor gamma-dependent adipocyte differentiation. Mol Cell Biol. 2009;29(18):5060-5069. doi: 10.1128/MCB.01001-08.

28. Dreijerink KM, Varier RA, van Nuland $R$, et al. Regulation of vitamin D receptor function in MEN1-related parathyroid adenomas. Mol Cell Endocrinol. 2009;313(1-2):1-8. doi: 10.1016/j.mce.2009.08.020.

29. Wu Y, Feng ZJ, Gao SB, et al. Interplay between menin and $\mathrm{K}$-Ras in regulating lung adenocarcinoma. J Biol Chem. 2012;287(47):40003-40011. doi: 10.1074/jbc.M112.382416. 
30. Feng ZJ, Gao SB, Wu Y, et al. Lung cancer cell migration is regulated via repressing growth factor PTN/RPTP $\beta / \zeta$ signaling by menin. Oncogene. 2010;29(39):5416-5426. doi: 10.1038/onc.2010.282.

31. Wang Y, Ozawa A, Zaman S, et al. The tumor suppressor protein menin inhibits AKT activation by regulating its cellular localization. Cancer Res. 2011;71(2):371-382. doi: 10.1158/0008-5472.CAN-10-3221.

32. Wuescher $L$, Angevine $K$, Hinds $T$, et al. Insulin regulates menin expression, cytoplasmic localization, and interaction with FOXO1. Am J Physiol Endocrinol Metab. 2011;301(3):E474-E483. doi: 10.1152/ajpendo.00022.2011.

33. Gurung B, Feng Z, Iwamoto DV, et al. Menin epigenetically represses Hedgehog signaling in MEN1 tumor syndrome. Cancer Res. 2013;73(8):2650-2658. doi: 10.1158/0008-5472.CAN-12-3158

34. Hughes E, Huang C. Participation of Akt, menin, and p21 in pregnancy-induced beta-cell proliferation. Endocrinology. 2011;152(3):847-855. doi: 10.1210/en.2010-1250.

35. Mensah-Osman E, Zavros Y, Merchant JL. Somatostatin stimulates menin gene expression by inhibiting protein kinase A. Am J Physiol Gastrointest Liver Physiol. 2008;295(4):G843-G854. doi: 10.1152/ajpgi.00607.2007.

36. Zhang H, Li W, Wang Q, et al. Glucose-mediated repression of menin promotes pancreatic $\beta$-cell proliferation. Endocrinology. 2012;153(2):602-611. doi: 10.1210/en.2011-1460.

37. Feng $Z, M a J$, Hua X. Epigenetic regulation by the menin pathway. Endocr Relat Cancer. 2017;24(10):T147-T159. doi: 10.1530/ERC-17-0298.

38. Iyer S, Agarwal SK. Epigenetic regulation in the tumorigenesis of MEN1-associated endocrine cell types. J Mol Endocrinol. 2018:61(1):R13-R24. doi: 10.1530/JME-18-0050.

39. Macfarlane LA, Murphy PR. MicroRNA: biogenesis, function and role in cancer. Curr Genomics. 2010;11(7):537-561. doi: 10.2174/138920210793175895.

40. Kung JT, Colognori D, Lee JT. Long noncoding RNAs: past, present, and future. Genetics. 2013;193(3):651-669. doi: 10.1534/genetics.112.146704

41. Di leva A, Butz H, Niamah M, et al. MicroRNAs as biomarkers in pituitary tumors. Neurosurgery. 2014;75(2):181-189. doi: 10.1227/NEU.0000000000000369.

42. Corbetta S, Vaira V, Guarnieri V, et al. Differential expression of microRNAs in human parathyroid carcinomas compared with normal parathyroid tissue. Endocr Relat Cancer. 2010;17(1):135-146. doi: 10.1677/ERC-09-0134

43. Szabó PM, Butz H, Igaz P, et al. Minireview: miRomics in endocrinology: a novel approach for modeling endocrine diseases. Mol Endocrinol. 2013;27(4):573-585. doi: 10.1210/me.2012-1220.

44. Herranz H, Cohen SM. MicroRNAs and gene regulatory networks: managing the impact of noise in biological systems. Genes Dev. 2010;24(13):1339-1344. doi: 10.1101/gad.1937010.

45. Luzi E, Marini F, Giusti F, et al. The negative feedback-loop between the oncomir Mir-24-1 and menin modulates the Men 1 tumorigenesis by mimicking the «Knudson's second hit». PLoS One. 2012;7(6):e39767. doi: 10.1371/journal.pone.0039767.

46. Vijayaraghavan J, Maggi EC, Crabtree JS. miR-24 regulates menin in the endocrine pancreas. Am J Physiol Endocrinol Metab. 2014;307(1):E84-92. doi: 10.1152/ajpendo.00542.2013.

47. Ehrlich L, Hall C, Venter J, et al. MiR-24 inhibition increases menin expression and decreases cholangiocarcinoma proliferation. Am J Pathol. 2017;187(3):570-580. doi: 10.1016/j.ajpath.2016.10.021.

48. Luzi E, Marini F, Ciuffi S, et al. An autoregulatory network between menin and pri-miR-24-1 is required for the processing of its specific modulator miR-24-1 in BON1 cells. Mol BioSyst. 2016;12(6):1922-1028. doi: 10.1039/c6mb00118a

49. Luzi E, Marini F, Tognarini I, et al. The regulatory network menin-microRNA 26a as a possible target for RNA-based therapy of bone diseases. Nucleic Acid Ther. 2012;22(2):103-108. doi: 10.1089/nat.2012.0344.

50. Li Y, Li W, Zhang JG, et al. Downregulation of tumor suppressor menin by miR-421 promotes proliferation and migration of neuroblastoma. Tumour Biol. 2014;35(10):10011-10017. doi: 10.1007/s13277-014-1921-1

51. LuY, Fei XQ, Yang SF, et al. Glucose-induced microRNA-17 promotes pancreatic beta cell proliferation through down-regulation of Menin Eur Rev Med Pharmacol Sci. 2015;19(4):624-629.

52. Hou R, Yang Z, Wang S, et al. miR-762 can negatively regulate menin in ovarian cancer. Onco Targets Ther. 2017;10:2127-2137. doi: $10.2147 / O T T . S 127872$

53. Gurung B, Muhammad AB, Hua X. Menin is required for optimal processing of the microRNA let-7a. J Biol Chem. 2014;289(14):9902-9908. doi: 10.1074/jbc.M113.520692.

54. Ouyang M, Su W, Xiao L, et al. MiR-29b modulates intestinal epithelium homeostasis by repressing menin translation. Biochem J. 2015;465(2):315-323. doi: 10.1042/BJ20141028.

55. Luzi E, Ciuffi S, Marini F, et al. Analysis of differentially expressed microRNAs in MEN1 parathyroid adenomas. Am J Trans/ Res. 2017;9(4):1743-1753.

56. Grolmusz VK, Borka K, Kövesdi A, et al. MEN1 mutations and potentially MEN1-targeting miRNAs are responsible for menin deficiency in sporadic and MEN1 syndrome-associated primary hyperparathyroidism. Virchows Arch. 2017;471(3):401-411. doi: 10.1007/s00428-017-2158-3.

57. Lines KE, Newey PJ, Yates CJ, et al. MiR-15a/miR-16-1 expression inversely correlates with cyclin D1 levels in Men 1 pituitary NETs. J Endocrinol. 2018;240(1):41-50. doi: 10.1530/JOE-18-0278.

58. Nagy Z, Szabó PM, Grolmusz VK, et al. MEN1 and microRNAs: The link between sporadic pituitary, parathyroid and adrenocortical tumors? Med Hypotheses. 2017:99:40-44. doi: 10.1016/j.mehy.2016.12.007.

59. Boyle B, Butz H, Liko I, et al. Expression of glucocorticoid receptor isoforms in human adrenocortical adenomas. Steroids. 2010;75(10):695-700. doi: 10.1016/j.steroids.2010.04.008.

60. Kopp F, Mendell JT. Functional classification and experimental dissection of Long Noncoding RNAs. Cell. 2018;172(3):393-407. doi: 10.1016/j.cell.2018.01.011.

61. Cao J. The functional role of long non-coding RNAs and epigenetics. Biol Proced Online. 2014;16:11. doi: 10.1186/1480-9222-16-11.

62. Modali SD, Parekh VI, Kebebew E, et al. Epigenetic regulation of the InCRNA MEG3 and its target C-MET in pancreatic neuroendocrine tumors. Mol Endocrinol. 2015;29(2):224-237. doi: 10.1210/me.2014-1304.

Рукопись получена: 29.04.2020. Одобрена к публикации: 15.06.2020. Опубликована online: 28.07.2020.

ИНФОРМАЦИЯ ОБ АВТОРАХ [AUTHORS INFO]

*Димитрова Диана Аршалуйсовна [Diana A. Dimitrova, M.D.]; адрес: Россия, 117036, г. Москва, ул. Дм. Ульянова, д. 11 [address: 11 Dm. Ulyanova street, 117036 Moscow, Russia];

ORCID: http://orcid.org/0000-0002-1359-8297; eLibrary SPIN: 5618-8971; e-mail: diadavtyan@gmail.com.

Мамедова Елизавета Октаевна, К.М.н. [Elizaveta O. Mamedova, MD, PhD]; e-mail: lilybet@mail.ru; ORCID: http://orcid.org/0000-0002-9783-3599; eLibrary SPIN: 3904-6017

Белая Жанна Евгеньевна, д.М.н., профессор [Zhanna E. Belaya, MD, PhD, Professor]; e-mail: jannabelaya@gmail.com; ORCID: http://orcid.org/0000-0002-6674-6441; eLibrary SPIN: 4746-7173

Мельниченко Галина Афанасьевна, д.м.н., профессор, академик PAH [Galina A. Melnichenko, MD, PhD, acad.]; eLibrary SPIN: 8615-0038; ORCID: https://orcid.org/0000-0002-5634-7877; e-mail: teofrast2000@mail.ru 


\section{ЦИТИРОВАТЬ:}

Мамедова Е.О., Димитрова Д.А., Белая Ж.Е., Мельниченко Г.А. Роль некодирующих РНК в патогенезе синдрома множественных эндокринных неоплазий 1 типа // Проблемы эндокринологии. - 2020. - Т. 66. — №2. — С.4-12. doi: https://doi.org/10.14341/probl12413

\section{TO CITE THIS ARTICLE:}

Mamedova EO, Dimitrova DA, Belaya ZE, Melnichenko GA The role of non-coding RNAs in the pathogenesis of multiple endocrine neoplasia type 1. Problems of Endocrinology. 2020;66(2):4-12. doi: https://doi.org/10.14341/probl12413 